\title{
On the diophantine equation $D_{1} x^{4}-D_{2} y^{2}=1$
}

\author{
by
}

\author{
MAOHUA LE (Zhanjiang)
}

1. Introduction. Let $\mathbb{Z}, \mathbb{N}, \mathbb{Q}, \mathbb{R}$ be the sets of integers, positive integers, rational numbers and real numbers respectively. Let $D_{1}, D_{2} \in \mathbb{N}$ with $\operatorname{gcd}\left(D_{1}, D_{2}\right)=1$. There were many papers concerned with the equation

$$
D_{1} x^{4}-D_{2} y^{2}=1, \quad x, y \in \mathbb{N},
$$

written by Ljunggren, Bumby, Cohn, Ke and Sun. Concerning the solvability of (1), Zhu [7] and Le [2] proved independently that if $D_{1}=1$, then (1) has solutions $(x, y)$ if and only if the fundamental solution $u_{1}+v_{1} \sqrt{D_{2}}$ of Pell's equation

$$
u^{2}-D_{2} v^{2}=1, \quad u, v \in \mathbb{Z},
$$

satisfies either $u_{1}=x_{1}^{2}$ or $u_{1}^{2}+D_{2} v_{1}^{2}=x_{1}^{2}$, where $x_{1} \in \mathbb{N}$. In addition, Zhu [7] showed that if $D_{2}=1$, then (1) has solutions $(x, y)$ if and only if the equation

$$
u^{\prime 2}-D_{1} v^{\prime 2}=-1, \quad u^{\prime}, v^{\prime} \in \mathbb{Z},
$$

has solutions $\left(u^{\prime}, v^{\prime}\right)$ and its least positive integer solution $\left(u_{1}^{\prime}, v_{1}^{\prime}\right)$ satisfies $v_{1}^{\prime}=x_{1}^{2}$, where $x_{1} \in \mathbb{N}$. In this paper we prove a general result as follows.

Theorem 1. If $\min \left(D_{1}, D_{2}\right)>1$, then (1) has solutions $(x, y)$ if and only if the equation

$$
D_{1} U^{2}-D_{2} V^{2}=1, \quad U, V \in \mathbb{Z},
$$

has solutions $(U, V)$ and its least positive integer solution $\left(U_{1}, V_{1}\right)$ satisfies $U_{1}=x_{1}^{2}$, where $x_{1} \in \mathbb{N}$.

Let $N\left(D_{1}, D_{2}\right)$ denote the number of solutions $(x, y)$ of (1). Ljunggren [4] showed that $N\left(1, D_{2}\right) \leq 2$. In [3], Le proved that if $D_{2}>e^{64}$, then $N\left(1, D_{2}\right) \leq 1$. Recently, Wu $[6]$ relaxed the condition $D_{2}>e^{64}$ to $D_{2}>e^{37}$. In this paper we prove the following result.

Supported by the National Natural Science Foundation of China and Guangdong Provincial Natural Science Foundation. 
THEOREM 2. If $D_{1}$ is a square and

$$
\max \left(D_{1}, D_{2}\right) \geq \begin{cases}9.379 \cdot 10^{8} & \text { if } \min \left(D_{1}, D_{2}\right)=1, \\ 2.374 \cdot 10^{10} & \text { if } \min \left(D_{1}, D_{2}\right)>1,\end{cases}
$$

then $N\left(D_{1}, D_{2}\right) \leq 1$.

\section{Preliminaries}

Lemma 1. For any odd prime $p$ with $p \equiv 1(\bmod 4)$, there exists $a_{1} \in \mathbb{N}$ such that $p>a_{1}>1,2 \nmid a_{1}$ and $\left(a_{1} / p\right)=-1$, where $\left(a_{1} / p\right)$ is Legendre's symbol.

Proof. It is a well known fact that there exists $a \in \mathbb{N}$ with $p>a>1$ and $(a / p)=-1$. Since $(a / p)=((p-a) / p)$ for $p \equiv 1(\bmod 4)$, we get

$$
a_{1}= \begin{cases}a & \text { if } 2 \nmid a, \\ p-a & \text { if } 2 \mid a .\end{cases}
$$

The lemma is proved.

Lemma 2 ([3, Lemma 3]). Let $d \in \mathbb{N}$ be square-free. If $(u, v)$ and $\left(u^{\prime}, v^{\prime}\right)$ are solutions of the equation

$$
u^{2}-d v^{2}=1, \quad u, v \in \mathbb{N},
$$

with $u^{\prime} \equiv 0(\bmod u)$, then there exist fixed $d_{1}, d_{2} \in \mathbb{N}$ such that

$$
\begin{gathered}
d_{1} d_{2}=d, \quad u+1=\delta d_{1} v_{1}^{2}, \quad u-1=\delta d_{2} v_{2}^{2}, \\
u^{\prime}+1=\delta d_{1} v_{1}^{\prime 2}, \quad u^{\prime}-1=\delta d_{2} v_{2}^{\prime 2},
\end{gathered}
$$

where $\delta, v_{1}, v_{2}, v_{1}^{\prime}, v_{2}^{\prime} \in \mathbb{N}$ satisfy

$$
\delta v_{1} v_{2}=v, \quad \delta v_{1}^{\prime} v_{2}^{\prime}=v^{\prime}, \quad \delta= \begin{cases}1 & \text { if } 2 \nmid v, \\ 2 & \text { if } 2 \mid v .\end{cases}
$$

Lemma 3 ([5]). For $\min \left(D_{1}, D_{2}\right)>1$, if $(2)$ has solutions $(U, V)$, then it has a unique positive integer solution $\left(U_{1}, V_{1}\right)$ such that $U_{1} \sqrt{D_{1}}+V_{1} \sqrt{D_{2}} \leq$ $U \sqrt{D_{1}}+V \sqrt{D_{2}}$ for all positive integer solutions $(U, V)$ of $(2) .\left(U_{1}, V_{1}\right)$ is called the least solution of (2). Moreover, all positive integer solutions $(U, V)$ of (2) are given by

$$
U \sqrt{D_{1}}+V \sqrt{D_{2}}=\left(U_{1} \sqrt{D_{1}}+V_{1} \sqrt{D_{2}}\right)^{t}, \quad t \in \mathbb{N}, 2 \nmid t .
$$

Lemma 4. For $\min \left(D_{1}, D_{2}\right)>1$, let $(U, V)$ be a solution of $(2)$, and let

$$
\varepsilon=U \sqrt{D_{1}}+V \sqrt{D_{2}}, \quad \bar{\varepsilon}=U \sqrt{D_{1}}-V \sqrt{D_{2}} .
$$

Further, for any $m \in \mathbb{Z}$ with $2 \nmid m$, let

$$
E(m)=\frac{\varepsilon^{m}+\bar{\varepsilon}^{m}}{\varepsilon+\bar{\varepsilon}} .
$$

Then $E(m) \in \mathbb{N}$ and: 
(i) $E(m)=E(-m)$.

(ii) $E(m) \equiv 1(\bmod 4), E(m) \equiv(-1)^{(m-1) / 2} m(\bmod U)$.

(iii) For any $m, m^{\prime} \in \mathbb{Z}$ with $2 \nmid m m^{\prime}, E(m) \equiv-E\left(m-2 m^{\prime}\right)\left(\bmod E\left(m^{\prime}\right)\right)$.

(iv) For any $m, m^{\prime} \in \mathbb{Z}$ with $2 \nmid m m^{\prime}$ and $\operatorname{gcd}\left(m, m^{\prime}\right)=1,\left(E(m) / E\left(m^{\prime}\right)\right)$ $=1$, where $(* / *)$ is the Jacobi symbol.

Proof. Since $\varepsilon \bar{\varepsilon}=1$ by (2) and (6), we get (i) by (7). Since we have $E(m+4)+E(m)=\left(\varepsilon^{2}+\bar{\varepsilon}^{2}\right) E(m+2) \equiv-2 E(m+2)(\bmod 4 U)$, (ii) follows by induction on $m$ in view of the fact that $E(-1)=E(1)=1$.

Notice that

$$
E(m)+E\left(m-2 m^{\prime}\right)=\left(\varepsilon^{m-m^{\prime}}+\bar{\varepsilon}^{m-m^{\prime}}\right) E\left(m^{\prime}\right),
$$

where $\varepsilon^{m-m^{\prime}}+\bar{\varepsilon}^{m-m^{\prime}} \in \mathbb{Z}$, since $m-m^{\prime}$ is even. So we have (iii). Moreover, using (8) and (ii), we obtain (iv) by induction. The lemma is proved.

By much the same argument as in the proof of Lemma 4, we can prove the following lemma.

Lemma 5. For $\min \left(D_{1}, D_{2}\right)>1$, let $U, V, \varepsilon, \bar{\varepsilon}$ be defined as in Lemma 4, and let

$$
F(m)=\frac{\varepsilon^{m}-\bar{\varepsilon}^{m}}{\varepsilon-\bar{\varepsilon}}
$$

for any $m \in \mathbb{Z}$ with $2 \nmid m$. Then $F(m) \in \mathbb{Z}$ and:

(i) $F(m)=-F(-m)$ and $F(m)>0$ if $m>0$.

(ii) For any $m, m^{\prime} \in \mathbb{Z}$ with $2 \nmid m m^{\prime}, F(m) \equiv F\left(m-2 m^{\prime}\right)(\bmod F(m))$.

LEMMA 6. If $(U, V)$ and $\left(U^{\prime}, V^{\prime}\right)$ are positive integer solutions of (2) satisfying $U^{\prime} \equiv 0(\bmod U)$ or $V^{\prime} \equiv 0(\bmod V)$, then there exists $t^{\prime} \in \mathbb{N}$ such that

$$
U^{\prime} \sqrt{D_{1}}+V^{\prime} \sqrt{D_{2}}=\left(U \sqrt{D_{1}}+V \sqrt{D_{2}}\right)^{t^{\prime}}, \quad 2 \nmid t^{\prime} .
$$

Proof. Let $\varepsilon=U_{1} \sqrt{D_{1}}+V_{1} \sqrt{D_{2}}$ and $\bar{\varepsilon}=U_{1} \sqrt{D_{1}}-V_{1} \sqrt{D_{2}}$, where $\left(U_{1}, V_{1}\right)$ is the least solution of $(2)$. By Lemma 3 , there exist $m, m^{\prime} \in \mathbb{N}$ such that

$$
U \sqrt{D_{1}}+V \sqrt{D_{2}}=\varepsilon^{m}, \quad U^{\prime} \sqrt{D_{1}}+V^{\prime} \sqrt{D_{2}}=\varepsilon^{m^{\prime}}, \quad 2 \nmid m m^{\prime} .
$$

Then we have $U=U_{1} E(m), V=V_{1} F(m), U^{\prime}=U_{1} E\left(m^{\prime}\right)$ and $V^{\prime}=$ $V_{1} F\left(m^{\prime}\right)$.

By Lemma 4(iii), if $U^{\prime} \equiv 0(\bmod U)$, then we have

$$
0 \equiv U^{\prime}=U_{1} E\left(m^{\prime}\right) \equiv-U_{1} E\left(m^{\prime}-2 m\right) \equiv \ldots \equiv \pm U_{1} E(s)(\bmod U),
$$

where $s \in \mathbb{Z}$ satisfies $2 \nmid s$ and $-m<s \leq m$. Since $0<E(s)<E(m)$ if $|s|<m$, we obtain $s=m$ by (11). Therefore, $m \mid m^{\prime}$ and (9) holds by (10). 
The proof in the case $V^{\prime} \equiv 0(\bmod V)$ is analogous. By Lemma 5(ii), we then have

$$
0 \equiv V^{\prime}=V_{1} F\left(m^{\prime}\right) \equiv V_{1} F\left(m^{\prime}-2 m\right) \equiv \ldots \equiv V_{1} F(s)(\bmod V),
$$

where $s \in \mathbb{Z}$ satisfies $2 \nmid s$ and $-m<s \leq m$. Since $0<|F(s)|<F(m)$ if $|s|<m$, we get $s=m$, and hence, $m \mid m^{\prime}$. Thus (9) holds in this case. The lemma is proved.

Let $\alpha$ be an algebraic number with the minimal polynomial

$$
a_{0} z^{d}+\ldots+a_{d}=a_{0} \prod_{i=1}^{d}\left(z-\sigma_{i} \alpha\right), \quad a_{0}>0,
$$

where $\sigma_{1} \alpha, \ldots, \sigma_{d} \alpha$ are all conjugates of $\alpha$. Then

$$
h(\alpha)=\frac{1}{d}\left(\log a_{0}+\sum_{i=1}^{d} \log \max \left(1,\left|\sigma_{i} \alpha\right|\right)\right)
$$

is called the logarithmic absolute height of $\alpha$.

LEMma 7 ([1, Corollary 2]). Let $\alpha_{1}, \alpha_{2}$ be real algebraic numbers with $\alpha_{1}>1$ and $\alpha_{2}>1$ which are multiplicatively independent, and let $\log A_{j} \geq \max \left(h\left(\alpha_{j}\right),\left|\log \alpha_{j}\right| / r, 1 / r\right)$ for $j=1,2$, where $r=\left[\mathbb{Q}\left(\alpha_{1}, \alpha_{2}\right)\right.$ : $\mathbb{Q}] /\left[\mathbb{R}\left(\alpha_{1}, \alpha_{2}\right): \mathbb{R}\right]$. If $\Lambda=b_{1} \log \alpha_{1}-b_{2} \log \alpha_{2} \neq 0$ for some $b_{1}, b_{2} \in \mathbb{N}$, then

$$
|\Lambda| \geq \exp \left(-24.34 r^{4}\left(\log A_{1}\right)\left(\log A_{2}\right)\left(\max \left(\log b^{\prime}+0.69,21 / r, 1 / 2\right)\right)^{2}\right),
$$

where $b^{\prime}=b_{1} /\left(r \log A_{2}\right)+b_{2} /\left(r \log A_{1}\right)$.

3. Proof of Theorem 1. The sufficiency of the theorem is clear; it suffices to prove the necessity. Now we assume that (1) has solutions $(x, y)$. Then (1) has a unique solution $\left(x_{1}, y_{1}\right)$ such that

$$
x_{1}^{2} \sqrt{D_{1}}+y_{1} \sqrt{D_{2}} \leq x^{2} \sqrt{D_{1}}+y \sqrt{D_{2}}
$$

for all solutions $(x, y)$ of $(1)$. Clearly, $\left(x_{1}^{2}, y_{1}\right)$ is a positive integer solution of $(2)$. Let $\left(U_{1}, V_{1}\right)$ be the least solution of $(2)$. By Lemma 3 , we have

$$
x_{1}^{2} \sqrt{D_{1}}+y_{1} \sqrt{D_{2}}=\left(U_{1} \sqrt{D_{1}}+V_{1} \sqrt{D_{2}}\right)^{t}, \quad t \in \mathbb{N}, 2 \nmid t .
$$

If $t=1$, then the theorem is proved. Otherwise, $t$ has an odd prime factor $p$. By Lemma $3,(2)$ has a positive integer solution $(U, V)$ which satisfies

$$
U \sqrt{D_{1}}+V \sqrt{D_{2}}=\left(U_{1} \sqrt{D_{1}}+V_{1} \sqrt{D_{2}}\right)^{t / p} .
$$

From (13) and (14), we get

$$
x_{1}^{2} \sqrt{D_{1}}+y_{1} \sqrt{D_{2}}=\left(U \sqrt{D_{1}}+V \sqrt{D_{2}}\right)^{p} .
$$


For any $m \in \mathbb{Z}$ with $2 \nmid m$, let $\varepsilon, \bar{\varepsilon}$ and $E(m)$ be defined as in (6) and (7), respectively. From (15) we get

$$
x_{1}^{2}=\frac{\varepsilon^{p}+\bar{\varepsilon}^{p}}{2 \sqrt{D_{1}}}=U E(p) .
$$

By Lemma 4(ii), $E(p) \in \mathbb{N}$ with $E(p) \equiv(-1)^{(p-1) / 2} p(\bmod U)$. This implies that $\operatorname{gcd}(U, E(p))=1$ or $p$.

If $\operatorname{gcd}(U, E(p))=1$, then from (16) we get $U=x_{11}^{2}$ and $E(p)=x_{12}^{2}$, where $x_{11}, x_{12} \in \mathbb{N}$ with $x_{11} x_{12}=x_{1}$. It follows that $\left(x_{11}, V\right)$ is a solution of (1) satisfying

$$
x_{11}^{2} \sqrt{D_{1}}+V \sqrt{D_{2}}=U \sqrt{D_{1}}+V \sqrt{D_{2}}=\varepsilon<\varepsilon^{p}=x_{1}^{2} \sqrt{D_{1}}+y_{1} \sqrt{D_{2}},
$$

which contradicts (12).

If $\operatorname{gcd}(U, E(p))=p$, then we have

$$
U=p x_{11}^{2}, \quad E(p)=p x_{12}^{2},
$$

where $x_{11}, x_{12} \in \mathbb{N}$ with $p x_{11} x_{12}=x_{1}$. Since $E(p) \equiv 1(\bmod 4)$ by Lemma 4(ii), we see from $(17)$ that $p \equiv 1(\bmod 4)$. Therefore, by Lemma 1 , there exists $a_{1} \in \mathbb{N}$ such that $2 \nmid a_{1}, p>a_{1}>1$ and $\left(a_{1} / p\right)=-1$. Further, since $p \mid U$, by Lemma 4(ii), we get $E\left(a_{1}\right) \equiv(-1)^{\left(a_{1}-1\right) / 2} a_{1}(\bmod p)$. So we have

$$
\begin{aligned}
\left(\frac{E(p)}{E\left(a_{1}\right)}\right) & =\left(\frac{p x_{12}^{2}}{E\left(a_{1}\right)}\right)=\left(\frac{p}{E\left(a_{1}\right)}\right)=\left(\frac{E\left(a_{1}\right)}{p}\right) \\
& =\left(\frac{(-1)^{\left(a_{1}-1\right) / 2} a_{1}}{p}\right)=\left(\frac{a_{1}}{p}\right)=-1,
\end{aligned}
$$

by (17). However, by Lemma 4(iv), (18) is impossible. The theorem is proved.

4. Proof of Theorem 2. First we consider the case where $\min \left(D_{1}, D_{2}\right)$ $>1$. By Theorem 1, if (1) has solutions $(x, y)$, then $\left(x_{1}, y_{1}\right)=\left(\sqrt{U_{1}}, V_{1}\right)$ is a solution of $(1)$, where $\left(U_{1}, V_{1}\right)$ is the least solution of (2). Further, by Lemma 3 , if $N\left(D_{1}, D_{2}\right)>1$, then (1) has another solution $\left(x_{2}, y_{2}\right)$ which satisfies $x_{2}>x_{1}$ and

$$
x_{2} \equiv 0\left(\bmod x_{1}\right) .
$$

Since $D_{1}$ is a square, $D_{2}$ cannot be such, therefore we may also assume, without loss of generality, that $D_{2}$ is square-free. Let $D_{1}=a^{2}$, where $a \in \mathbb{N}$ with $a>1$. Then $\left(a x_{1}^{2}, y_{1}\right)$ and $\left(a x_{2}^{2}, y_{2}\right)$ are solutions of the equation

$$
u^{2}-D_{2} v^{2}=1, \quad u, v \in \mathbb{N} .
$$

Notice that $a x_{2}^{2} \equiv 0\left(\bmod a x_{1}^{2}\right)$ by $(19)$. We have

$$
\begin{array}{ll}
a x_{1}^{2}+1=\delta D_{21} y_{11}^{2}, & a x_{1}^{2}-1=\delta D_{22} y_{12}^{2}, \\
a x_{2}^{2}+1=\delta D_{21} y_{21}^{2}, & a x_{2}^{2}-1=\delta D_{22} y_{22}^{2},
\end{array}
$$


by Lemma 2 , where $\delta, D_{21}, D_{22}, y_{11}, y_{12}, y_{21}, y_{22} \in \mathbb{N}$ satisfy

$$
D_{21} D_{22}=D_{2}, \quad \operatorname{gcd}\left(D_{21}, D_{22}\right)=1,
$$

$$
\delta y_{11} y_{12}=y_{1}, \quad \delta y_{21} y_{22}=y_{2}, \quad \delta= \begin{cases}1 & \text { if } 2 \nmid y_{1}, \\ 2 & \text { if } 2 \mid y_{1}\end{cases}
$$

We see from (20) and (21) that $\left(y_{11}, x_{1}\right)$ and $\left(y_{21}, x_{2}\right)$ are solutions of the equation

$$
\delta D_{21} X^{2}-a Y^{2}=1, \quad X, Y \in \mathbb{N},
$$

while $\left(x_{1}, y_{12}\right)$ and $\left(x_{2}, y_{22}\right)$ are solutions of the equation

$$
a X^{\prime 2}-\delta D_{22} Y^{\prime 2}=1, \quad X^{\prime}, Y^{\prime} \in \mathbb{N} .
$$

Let

$$
\begin{array}{ll}
\varepsilon_{1}=x_{1} \sqrt{a}+y_{11} \sqrt{\delta D_{21}}, & \bar{\varepsilon}_{1}=x_{1} \sqrt{a}-y_{11} \sqrt{\delta D_{21}}, \\
\varepsilon_{2}=x_{1} \sqrt{a}+y_{12} \sqrt{\delta D_{22}}, & \bar{\varepsilon}_{2}=x_{1} \sqrt{a}-y_{12} \sqrt{\delta D_{22}} .
\end{array}
$$

Recall that $x_{2} \equiv 0\left(\bmod x_{1}\right)$ by $(19)$. Using Lemma 6 , we have

$$
\begin{array}{ll}
x_{2} \sqrt{a}+y_{21} \sqrt{\delta D_{21}}=\varepsilon_{1}^{t_{1}}, & x_{2} \sqrt{a}-y_{21} \sqrt{\delta D_{21}}=\bar{\varepsilon}_{1}^{t_{1}}, \\
x_{2} \sqrt{a}+y_{22} \sqrt{\delta D_{22}}=\varepsilon_{2}^{t_{2}}, & x_{2} \sqrt{a}-y_{22} \sqrt{\delta D_{22}}=\bar{\varepsilon}_{2}^{t_{2}},
\end{array}
$$

where $t_{1}, t_{2} \in \mathbb{N}$ satisfy $t_{1}>1, t_{2}>1$ and $2 \nmid t_{1} t_{2}$. From (24)-(27), we obtain

$$
\begin{gathered}
\varepsilon_{1}+\bar{\varepsilon}_{1}=\varepsilon_{2}+\bar{\varepsilon}_{2}, \\
\varepsilon_{1}^{t_{1}}+\bar{\varepsilon}_{1}^{t_{1}}=\varepsilon_{2}^{t_{2}}+\bar{\varepsilon}_{2}^{t_{2}} .
\end{gathered}
$$

Let $\Delta=\bar{\varepsilon}_{2}-\bar{\varepsilon}_{1}$ and $\Delta^{\prime}=\bar{\varepsilon}_{2}^{t_{2}}-\bar{\varepsilon}_{1}^{t_{1}}$. Since $\varepsilon_{1} \bar{\varepsilon}_{1}=-1$ and $\varepsilon_{2} \bar{\varepsilon}_{2}=1$, from (28) and (29) we get

$$
\begin{aligned}
\log \varepsilon_{1} & =\log \varepsilon_{2}+\frac{2 \Delta}{\varepsilon_{1}+\varepsilon_{2}} \sum_{i=0}^{\infty} \frac{1}{2 i+1}\left(\frac{\Delta}{\varepsilon_{1}+\varepsilon_{2}}\right)^{2 i} \\
& =\log \varepsilon_{2}+\frac{2}{\varepsilon_{1} \varepsilon_{2}} \sum_{i=0}^{\infty} \frac{1}{2 i+1}\left(\frac{1}{\varepsilon_{1} \varepsilon_{2}}\right)^{2 i} \\
& =\log \varepsilon_{2}+\frac{2}{\varepsilon_{2}^{2}}\left(\frac{\varepsilon_{2}}{\varepsilon_{1}} \sum_{i=0}^{\infty} \frac{1}{2 i+1}\left(\frac{1}{\varepsilon_{1} \varepsilon_{2}}\right)^{2 i}\right) \\
& =\log \varepsilon_{2}+\frac{2}{\varepsilon_{2}^{2}}\left(\frac{1}{1+1 /\left(\varepsilon_{1} \varepsilon_{2}\right)+1 / \varepsilon_{2}^{2}} \sum_{i=0}^{\infty} \frac{1}{2 i+1}\left(\frac{1}{\varepsilon_{1} \varepsilon_{2}}\right)^{2 i}\right) \\
& <\log \varepsilon_{2}+\frac{2}{\varepsilon_{2}^{2}}, \\
\text { (31) } t_{1} \log \varepsilon_{1} & =t_{2} \log \varepsilon_{2}+\frac{2 \Delta^{\prime}}{\varepsilon_{1}^{t_{1}}+\varepsilon_{2}^{t_{2}}} \sum_{i=0}^{\infty} \frac{1}{2 i+1}\left(\frac{\Delta^{\prime}}{\varepsilon_{1}^{t_{1}}+\varepsilon_{2}^{t_{2}}}\right)^{2 i}
\end{aligned}
$$




$$
\begin{aligned}
& =t_{2} \log \varepsilon_{2}+\frac{2}{\varepsilon_{1}^{t_{1}} \varepsilon_{2}^{t_{2}}} \sum_{i=0}^{\infty} \frac{1}{2 i+1}\left(\frac{1}{\varepsilon_{1}^{t_{1}} \varepsilon_{2}^{t_{2}}}\right)^{2 i} \\
& =t_{2} \log \varepsilon_{2}+\frac{2}{\varepsilon_{2}^{2 t_{2}}}\left(\frac{\varepsilon_{2}^{t_{2}}}{\varepsilon_{1}^{t_{1}}} \sum_{i=0}^{\infty} \frac{1}{2 i+1}\left(\frac{1}{\varepsilon_{1}^{t_{1}} \varepsilon_{2}^{t_{2}}}\right)^{2 i}\right) \\
& =t_{2} \log \varepsilon_{2}+\frac{2}{\varepsilon_{2}^{2 t_{2}}}\left(\frac{1}{1+1 /\left(\varepsilon_{1}^{t_{1}} \varepsilon_{2}^{t_{2}}\right)+1 / \varepsilon_{2}^{2 t_{2}}} \sum_{i=0}^{\infty} \frac{1}{2 i+1}\left(\frac{1}{\varepsilon_{1}^{t_{1}} \varepsilon_{2}^{t_{2}}}\right)^{2 i}\right) \\
& <t_{2} \log \varepsilon_{2}+\frac{2}{\varepsilon_{2}^{2 t_{2}}},
\end{aligned}
$$

respectively. By (30) and (31), we get $\log \varepsilon_{1}-\log \varepsilon_{2}>t_{1} \log \varepsilon_{1}-t_{2} \log \varepsilon_{2}>0$. This implies that $\left(t_{2}-1\right) \log \varepsilon_{2}>\left(t_{1}-1\right) \log \varepsilon_{1}$. Since $\varepsilon_{1}>\varepsilon_{2}>1$ by $(28)$, we obtain $t_{2}>t_{1}$. Since $2 \nmid t_{1} t_{2}$, we get

$$
t_{2} \geq t_{1}+2 \text {. }
$$

Therefore, we find from (30)-(32) that

$$
t_{2}>\frac{\left(t_{2}-t_{1}\right) \log \varepsilon_{1}}{\log \varepsilon_{1}-\log \varepsilon_{2}}>\varepsilon_{2}^{2} \log \varepsilon_{1}>\varepsilon_{1}^{2}\left(\log \varepsilon_{1}\right) e^{-4 / \varepsilon_{2}^{2}} .
$$

Let $K_{1}=\mathbb{Q}\left(\sqrt{\delta D_{21} a}\right)$ and $K_{2}=\mathbb{Q}\left(\sqrt{\delta D_{22} a}\right)$. Since $D_{2}$ is not a square, we see from (22) that $K_{1} \backslash \mathbb{Q} \cap K_{2} \backslash \mathbb{Q}=\emptyset$. If there exist $k_{1}, k_{2} \in \mathbb{Q}$ such that $\varepsilon_{1}^{k_{1}} \varepsilon_{2}^{k_{2}}=1$, then $\varepsilon_{1}^{m_{1}} \varepsilon_{2}^{m_{2}}=1$ for some $m_{1}, m_{2} \in \mathbb{Z}$ with $2 \mid m_{1}$ and $2 \mid m_{2}$. Notice that $\varepsilon_{1}^{m} \in K_{1} \backslash \mathbb{Q}$ and $\varepsilon_{2}^{m} \in K_{2} \backslash \mathbb{Q}$ for any $m \in \mathbb{Z} \backslash\{0\}$ with $2 \mid m$. We get $m_{1}=m_{2}=0$ and $k_{1}=k_{2}=0$. This implies that $\varepsilon_{1}$ and $\varepsilon_{2}$ are multiplicatively independent.

Let $h\left(\varepsilon_{1}\right), h\left(\varepsilon_{2}\right)$ denote the logarithmic absolute heights of $\varepsilon_{1}, \varepsilon_{2}$ respectively, and let $r$ denote the degree of $\mathbb{Q}\left(\varepsilon_{1}, \varepsilon_{2}\right)$. Then

$$
4 \leq r \leq 8, \quad h\left(\varepsilon_{1}\right)=\frac{\log \varepsilon_{1}}{r / 2}, \quad h\left(\varepsilon_{2}\right)=\frac{\log \varepsilon_{2}}{r / 2} .
$$

Further, let $\Lambda=t_{1} \log \varepsilon_{1}-t_{2} \log \varepsilon_{2}$ and

$$
t=\frac{t_{1}}{2 \log \varepsilon_{2}}+\frac{t_{2}}{2 \log \varepsilon_{1}} .
$$

Then we have

$$
t=\frac{t_{2}}{\log \varepsilon_{1}}+\frac{\Lambda}{2\left(\log \varepsilon_{1}\right)\left(\log \varepsilon_{2}\right)}<\frac{t_{2}}{\log \varepsilon_{1}}\left(1+\frac{1}{t_{2} \varepsilon_{2}^{2 t_{2}} \log \varepsilon_{2}}\right),
$$

by (31). Using Lemma 7, from (34) we get

$$
\Lambda \geq \exp \left(-6232\left(\log \varepsilon_{1}\right)\left(\log \varepsilon_{2}\right)(\max (\log t+0.69,21 / 4))^{2}\right) .
$$


We now suppose that $\varepsilon_{1} \geq 785$. By $(28), \varepsilon_{1}^{2}-\left(\varepsilon_{2}+\bar{\varepsilon}_{2}\right) \varepsilon_{1}-1=0$. Since $\varepsilon_{2} \geq 1+\sqrt{2}$, we get $\varepsilon_{1}<1.745 \varepsilon_{2}$, whence $\varepsilon_{2}>449.856$. If $\log t+0.69 \leq 21 / 4$, then $t<96$ and

$$
t_{2}<96 \log \varepsilon_{1},
$$

by (35). The combination of (33) and (38) yields $96>\varepsilon_{1}^{2} e^{-4 / \varepsilon_{2}^{2}}>6 \cdot 10^{5}$, a contradiction. Hence, $\log t+0.69>21 / 4$, and

$$
\Lambda \geq \exp \left(-6232\left(\log \varepsilon_{1}\right)\left(\log \varepsilon_{2}\right)(\log t+0.69)^{2}\right),
$$

by (37). The combination of (31) and (39) yields

$$
\log 2+6232\left(\log \varepsilon_{1}\right)\left(\log \varepsilon_{2}\right)(\log t+0.69)^{2}>2 t_{2} \log \varepsilon_{2} .
$$

Further, by (31), (36) and (40), we get

$$
\begin{aligned}
1+ & 3116\left(\log \left(\frac{t_{2}}{\log \varepsilon_{1}}\right)+0.7\right)^{2} \\
& >\frac{\log 2}{\log \varepsilon_{2}}+3116\left(\log \left(\frac{t_{2}}{\log \varepsilon_{1}}+\frac{\Lambda}{2\left(\log \varepsilon_{1}\right)\left(\log \varepsilon_{2}\right)}\right)+0.69\right)^{2}>\frac{t_{2}}{\log \varepsilon_{1}},
\end{aligned}
$$

whence we conclude that

$$
\frac{t_{2}}{\log \varepsilon_{1}}<615000 .
$$

Therefore, from (33) and (41) we get $616000<\varepsilon_{1}^{2} e^{-4 / \varepsilon_{2}^{2}}<615000$, a contradiction. So we have

$$
\varepsilon_{1}<785
$$

From (22), (24), (25) and (28), we get

$$
\varepsilon_{1}=\varepsilon_{2}-\bar{\varepsilon}_{1}+\bar{\varepsilon}_{2}>\varepsilon_{2}+\bar{\varepsilon}_{2}=2 x_{1} \sqrt{a} \geq 2 \sqrt{a}=2 D_{1}^{1 / 4}
$$

and

$$
\begin{aligned}
\varepsilon_{1}^{2} & >\varepsilon_{1} \varepsilon_{2}-\varepsilon_{1} / \varepsilon_{2}+\varepsilon_{2} / \varepsilon_{1}-1 /\left(\varepsilon_{1} \varepsilon_{2}\right)=\left(\varepsilon_{1}-\bar{\varepsilon}_{1}\right)\left(\varepsilon_{2}-\bar{\varepsilon}_{2}\right) \\
& =\left(2 y_{11} \sqrt{\delta D_{21}}\right)\left(2 y_{12} \sqrt{\delta D_{22}}\right) \geq 4 \sqrt{D_{21} D_{22}}=4 \sqrt{D_{2}} .
\end{aligned}
$$

Therefore, by (42)-(44), we obtain $\max \left(D_{1}, D_{2}\right)<\varepsilon_{1}^{4} / 16<2.374 \cdot 10^{10}$. Thus, if $\min \left(D_{1}, D_{2}\right)>1$ and $\max \left(D_{1}, D_{2}\right) \geq 2.374 \cdot 10^{10}$, then $N\left(D_{1}, D_{2}\right)$ $\leq 1$.

Next we consider the case where $\min \left(D_{1}, D_{2}\right)=1$. Since $D_{1}$ is a square, we have $D_{1}=1$ and $D_{2}>1$. By much the same argument as in the proof of the case $\min \left(D_{1}, D_{2}\right)>1$, we can find from the proof of $[3$, Theorem 1] that if $D_{2} \neq 1785$ and $N\left(1, D_{2}\right)>1$, then there exist $t_{1}, t_{2} \in \mathbb{N}$ and real quadratic algebraic numbers $\varrho_{1}, \varrho_{2}$ satisfying $1<t_{1}<t_{2}, 1<\varrho_{2}<\varrho_{1}$, $D_{2}<\varrho_{1}^{4} / 16, h\left(\varrho_{1}\right)=\left(\log \varrho_{1}\right) / 2, h\left(\varrho_{2}\right)=\left(\log \varrho_{2}\right) / 2,\left[\mathbb{Q}\left(\varrho_{1}, \varrho_{2}\right): \mathbb{Q}\right]=4$,

(45) $0<\log \varrho_{1}-\log \varrho_{2}<2 / \varrho_{2}^{2}, \quad 0<t_{1} \log \varrho_{1}-t_{2} \log \varrho_{2}<2 / \varrho_{2}^{2 t_{2}}$, 


$$
t_{2}>\varrho_{1}^{2}\left(\log \varrho_{1}\right) e^{-4 / \varrho_{2}^{2}} .
$$

Using Lemma 7, we get either $\varrho_{1}^{2} e^{-4 / \varrho_{2}^{2}}<96$ or

$$
t_{1} \log \varrho_{1}-t_{2} \log \varrho_{2} \geq \exp \left(-1558\left(\log \varrho_{1}\right)\left(\log \varrho_{2}\right)(\log t+0.69)^{2}\right),
$$

where

$$
\begin{aligned}
t & =\frac{t_{1}}{2 \log \varrho_{2}}+\frac{t_{2}}{2 \log \varrho_{1}}=\frac{t_{2}}{\log \varrho_{1}}+\frac{t_{1} \log \varrho_{1}-t_{2} \log \varrho_{2}}{2\left(\log \varrho_{1}\right)\left(\log \varrho_{2}\right)} \\
& <\frac{t_{2}}{\log \varrho_{1}}+\frac{1}{2 \varrho_{2}^{2 t_{2}}\left(\log \varrho_{1}\right)\left(\log \varrho_{2}\right)} .
\end{aligned}
$$

We now suppose that $\varrho_{1} \geq 350$. Then from (45), (47) and (48) we get

$$
t_{2} / \log \varrho_{1}<122000 \text {. }
$$

The combination of (46) and (49) yields $122400<\varrho_{1}^{2} e^{-4 / \varrho_{2}^{2}}<122000$, a contradiction. So we have $\varrho_{1}<350$ and $D_{2}<9.379 \cdot 10^{8}$. This implies that if $\min \left(D_{1}, D_{2}\right)=1$ and $\max \left(D_{1}, D_{2}\right) \geq 9.379 \cdot 10^{8}$, then $N\left(D_{1}, D_{2}\right) \leq 1$. The proof is complete.

Acknowledgements. The author is grateful to the referees for their valuable suggestions.

\section{References}

[1] M. Laurent, M. Mignotte et Y. Nesterenko, Formes linéaires en deux logarithmes et déterminants d'interpolation, J. Number Theory 55 (1995), 285-321.

[2] M.-H. Le, A necessary and sufficient condition for the equation $x^{4}-D y^{2}=1$ to having positive integer solutions, Chinese Sci. Bull. 30 (1985), 1698.

[3] - A note on the diophantine equation $x^{2 p}-D y^{2}=1$, Proc. Amer. Math. Soc. 107 (1989), 27-34.

[4] W. Ljunggren, Über die Gleichung $x^{4}-D y^{2}=1$, Arch. Math. Naturv. 45 (5) (1942), 61-70.

[5] K. Petr, Sur l'équation de Pell, Časopis Pest. Mat. Fys. 56 (1927), 57-66 (in Czech).

[6] H.-M. Wu, On the number of solutions of the diophantine equation $x^{4}-D y^{2}=1$, J. Zhanjiang Teachers College Nat. Sci. 1995 (1), 12-15 (in Chinese).

[7] W.-S. Zhu, The solvability of equation $x^{4}-D y^{2}=1$, Acta Math. Sinica 28 (1985), 681-683 (in Chinese).

Department of Mathematics Zhanjiang Teachers College

P.O. Box 524048

Zhanjiang, Guangdong, P.R. China 